\section{Blinking and superficial punctate keratopathy in patients with diabetes mellitus}

${ }^{1}$ Inouye Eye Hospital

Tokyo, Japan

${ }^{2}$ Department of Ophthalmology The University of Tokyo Graduate School of

Medicine, Tokyo, Japan

${ }^{3}$ Department of Ophthalmology, University of Tsukuba, Ibaragi, Japan

${ }^{4}$ Department of Ophthalmology, Tokyo Women's Medical College Tokyo, Japan

${ }^{5}$ Taisho Pharmaceutical Co. Ltd, Saitama, Japan

Correspondence: $\mathrm{K}$ Inoue Inouye Eye Hospital, 4-3 Kanda-Surugadai, Chiyodaku, Tokyo 101-0062, Japan Tel: + 81332950911

Fax: + 81332950917

E-mail: inoue-k@

fd5.so-net.ne.jp

Received: 22 September 2003

Accepted in revised form: 2 February 2004 Published online: 30 July 2004
Abstract

Aim To evaluate blinking patterns in patients with diabetes mellitus and whether blinking contributes to the formation of superficial punctate keratopathy in diabetic patients. Methods We examined 163 patients with type II diabetes mellitus and 76 without diabetes. Blinks were recorded, analysed using six parameters, and compared between patients with and without diabetes. Multivariate regression analysis was performed to assess the influence of other ocular factors, such as status of tear lipid layer, tear breakup time, corneal sensitivity, the result of cotton thread test, or blinking rate related to superficial punctate keratopathy.

Results In patients with diabetes, the average mean and maximum interblinking times were longer, the average coefficient of variation of interblinking time was higher, and the average blinking rates were lower than those in patients without diabetes. Multivariate regression analysis revealed that the status of tear lipid layer and tear breakup time were significantly relevant to superficial punctate keratopathy $(P<0.01)$.

Conclusion Interblinking time was longer in diabetic patients, resulting in a decreased blinking rate. The prevalence of superficial punctate keratopathy cannot be predicted from blinking patterns in patients with diabetes.

Eye (2005) 19, 418-421. doi:10.1038/sj.eye.6701497 Published online 30 July 2004

Keywords: blinking; superficial punctate keratopathy; diabetes mellitus

\section{Introduction}

Diabetes mellitus is a syndrome characterized by hyperglycemia leading to associated
K Inoue ${ }^{1,2}$, K Okugawa $^{2}$, S Amano ${ }^{2}$, T Oshika ${ }^{3}$ E Takamura ${ }^{4}$, F Egami ${ }^{5}$, G Umizu ${ }^{5}$, K Aikawa ${ }^{5}$ and $\mathrm{S} \mathrm{Kato}^{2}$ microvascular and/or macrovascular complications. Patients with this disease often develop not only diabetic retinopathy but also keratoepitheliopathies, such as superficial punctate keratopathy, recurrent corneal erosion, and persistent epithelial defects. ${ }^{1-4}$ Diabetic keratoepitheliopathy has been attributed to quantitative and qualitative abnormalities in tear secretion, ${ }^{1}$ decreased corneal sensitivity, ${ }^{1,2}$ and poor adhesion of regenerating epithelial cells. ${ }^{3}$ We also found that qualitative abnormalities in tear secretion such as nonuniformity of the tear lipid layer and decreased tear breakup time, and corneal sensitivity were possibly relevant to diabetic keratoepitheliopathy. ${ }^{5}$

Furthermore, we hypothesized that diabetic patients may show changes in blinking patterns that would affect keratoepitheliopathy. There have been, however, no previous reports evaluating blinking patterns in patients with diabetes or the relationship between diabetic keratoepitheliopathy and blinking.

The aims of this study were to evaluate patterns of blinking in patients with diabetes mellitus and whether blinking contributed to the formation of keratoepitheliopathy, especially superficial punctate keratopathy in diabetic patients.

\section{Materials and methods}

We examined 163 patients (97 men and 66 women) with type II diabetes mellitus at Nadogaya Hospital (Chiba, Japan) between January and March of 2002. The mean patient age was $65.6 \pm 9.7$ years (mean \pm standard deviation) (range $45-84$ years). The mean duration of diabetes mellitus was $10.2 \pm 8.7$ years (range 6 months -40 years). In all, 93 patients were without diabetic retinopathy, 62 with nonproliferative retinopathy, and eight 
with proliferative retinopathy. The mean value of haemoglobin A1c was 7.3 $\pm 1.6 \%$ (range 4.7-13.3\%) at the time of examination. Patients who had intraocular surgery before were excluded from this study. As for controls, 76 patients (33 men and 43 women) with cataract were examined. These controls did not have diabetes mellitus without intraocular surgery and abnormalities of the cornea, conjunctiva, or eyelid. Patients whose chief complaints were dryness were excluded. The mean control patient age was $68.1 \pm 9.3$ years (range 33-81 years). All patients in both groups had a corrected visual acuity of 20/200 or better. There was not much difference in age between the diabetic and control groups. All patients in both groups were provided with informed consent to participate in the study.

The severity of corneal surface damage (superficial punctate keratopathy) was evaluated by staining the cornea with fluorescein, and the area and density of staining were recorded. ${ }^{6,7}$ The product of area $(0,1,2$, or 3 ) and density $(0,1,2$, or 3$)$ was used as the index of corneal surface damage (Table 1 ).

Measurements and the blinking analysis based on the previous report ${ }^{8}$ were performed. The patients were allowed to relax in a chair, and were asked to look at a videotape camera placed $3 \mathrm{~m}$ away from the patient. They were videotaped for at least $3 \mathrm{~min}$, with the middle $90 \mathrm{~s}$ used for analysis. The recorded images were analysed, using a blink analyser, for each patient. The blink analyser calculated the following six parameters: (1) mean interblinking time (IBT) - the mean time from the end of the blink to the start of the next blink, (2) mean blinking time (BT) - the mean time from the start to the end of one blink, (3) Max IBT-the longest observed IBT during measurement, (4) Max BT-the longest observed BT during measurement, (5) coefficient of variation $(\mathrm{CV})$ of IBT - the SD of the mean IBT divided by the mean IBT (this reflects the irregularity of the blinking pattern), and (6) CV of BT - the SD of the mean BT divided by the mean BT (this reflects the irregularity of the blink itself).

Tear film status was assessed using the specular reflection videorecording system (DR-1, Kowa Co., Tokyo, Japan). ${ }^{7}$ The DR-1 camera focuses on a $2.2 \times 3.0 \mathrm{~mm}^{2}$ area of the central cornea. The obtained

Table 1 Classification of severity corneal epithelial lesions

\begin{tabular}{ll}
\hline Area: corneal surface area & Density: density of damaged lesions \\
\hline A0: no punctate staining & D0: no punctate staining \\
A1: less than one-third & D1: sparse density \\
A2: one-third to two-thirds & D2: moderate density \\
A3: more than two-thirds & D3: high density with \\
& overlapping lesions \\
\hline
\end{tabular}

Table 2 Classification of tear lipid layer interference patterns

Grade 1: greyish colour and uniform duistribution

Grade 2: greyish colour with uneven distribution

Grade 3: two or more colours, uneven distribution

Grade 4: numerous colours

Grade 5: visible corneal surface

images were printed out and the status of lipid layer was classified into five grades based on the specific interference colour (Table 2). ${ }^{7}$ The cotton thread test was performed according to standard procedures. ${ }^{9}$ The thread was placed on the palpebral conjunctiva at the lateral one-fifth of the lower lid. After $15 \mathrm{~s}$, the thread was gently removed and the amount of tear secretion was measured. Tear breakup time was measured as the time between the last complete blink and the first disturbance of the precorneal tear film. ${ }^{10}$ The corneal sensitivity was measured by means of a Cochet-Bonnet aesthesiometer. ${ }^{11}$ The tip of the fully extended Cochet-Bonnet aesthesiometer was advanced steadily toward the centre of cornea. When the end plate of the aesthesiometer fibre was found to be in contact with the surface of the cornea, slight pressure was exerted just to visibly bend the fibre, starting at $6.0 \mathrm{~cm}$ and progressing by $0.5 \mathrm{~cm}$ decrements. The right eye of each patient was used in this study.

The unpaired $t$ test or the Mann-Whitney $U$-test was used to compare the diabetic and control groups. Multivariate regression analysis was performed in diabetic patients to determine how much variance in superficial punctate keratopathy was explained by the measured variables. The blinking rate, lipid layer status, tear volume, corneal sensitivity, and tear breakup time were used as independent variables. The dependent variable was the index of superficial punctate keratopathy (the area grade $\times$ density grade).

\section{Results}

In patients without diabetes, the overall average of the mean IBT was $5.5 \pm 5.9 \mathrm{~s}$, with an average Max IBT of $13.5 \pm 11.7 \mathrm{~s}$ and an average CV of IBT of $71 \pm 32 \%$

(Figure 1). The average mean BT was $0.19 \pm 0.05 \mathrm{~s}$, with an average Max BT of $0.34 \pm 0.24 \mathrm{~s}$ and an average CV of BT of $22 \pm 15 \%$. In $1 \mathrm{~min}, 22.2 \pm 18.5$ blinks were observed. In patients with diabetes, the overall average of the mean IBT was $7.5 \pm 8.0 \mathrm{~s}(P=0.04$, Mann-Whitney test), with an average Max IBT of $18.1 \pm 14.5 \mathrm{~s}(P=0.006$, MannWhitney test) and an average CV of IBT of $83 \pm 35 \%$ $(P=0.02, t$-test $)$. The average mean BT was $0.20 \pm 0.05 \mathrm{~s}$ $(P=0.35, t$-test $)$, with an average Max BT of $0.39 \pm 0.38 \mathrm{~s}$ $(P=0.62$, Mann-Whitney test $)$ and an average $C V$ of BT of $25 \pm 20 \%$ ( $P=0.38$, Mann-Whitney test). In $1 \mathrm{~min}$, 

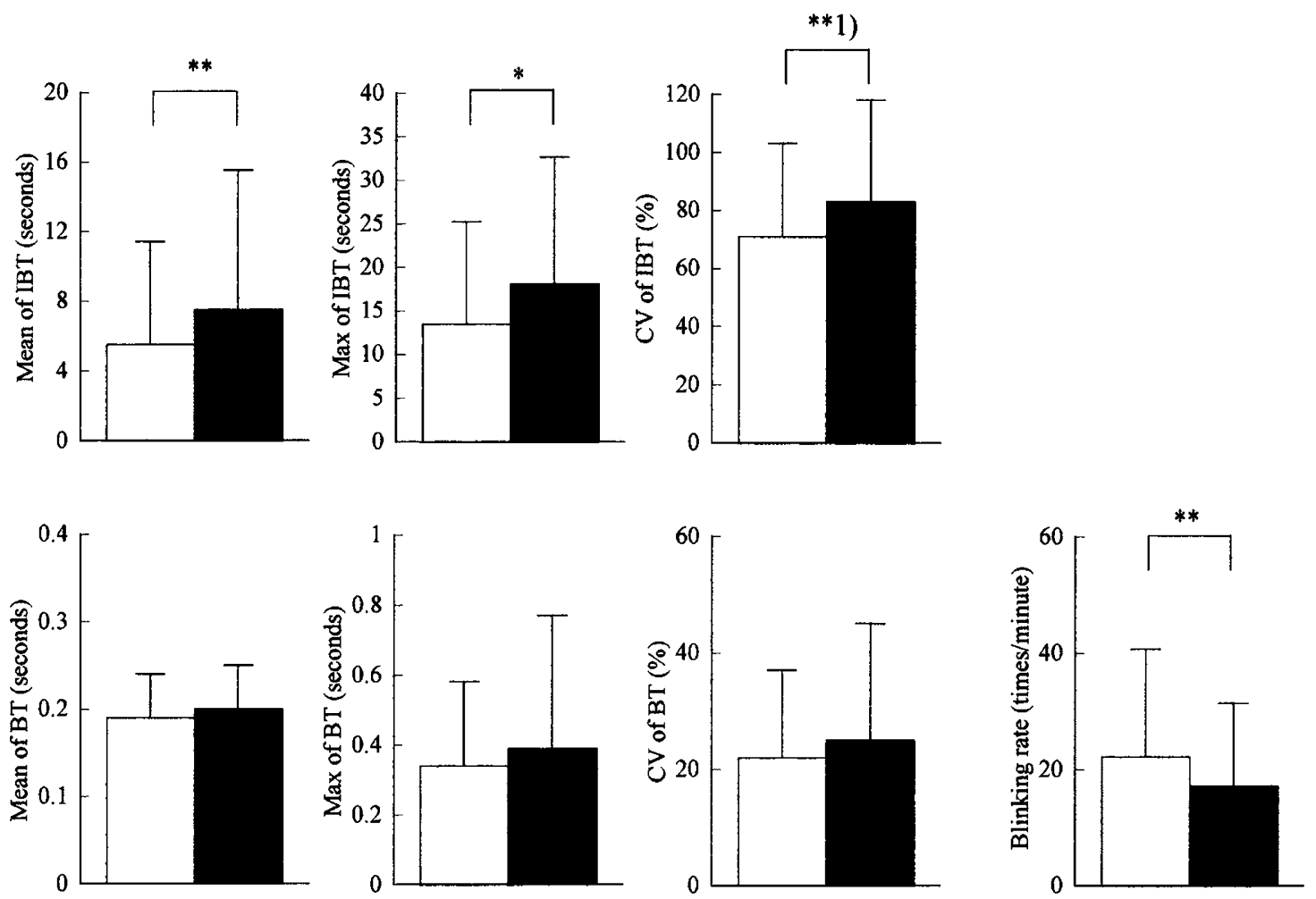

Figure 1 Blinking parameters (Mean of IBT, Max of IBT, CV of IBT, Mean of BT, Max of BT, and CV of BT) and blinking rates in patients without diabetes $(\square)$ and those with diabetes $(\square)$ (Mann-Whitney test; ${ }^{*} P<0.01,{ }^{* *} P<0.05$, unpaired $t$-test; ${ }^{* * 1} P<0.05$ ).

17.1 \pm 14.3 blinks $(P=0.04$, Mann-Whitney test $)$ were observed.

Multivariate regression analysis showed that the status of tear lipid layer and tear breakup time were significantly correlated with the index of superficial punctate keratopathy $\left(P<0.0001, R^{2}=0.36\right)$ (Table 3$)$. The blinking rate $(P=0.58)$, the results of corneal sensitivity $(P=0.62)$, and cotton thread test $(P=0.70)$ were not significantly correlated with the index of superficial punctate keratopathy.

\section{Discussion}

Blinking plays a role in maintaining ocular surface moisture and to wash foreign bodies out of the eyes with tears. Blinking rates reportedly range from 14.3 to 24 per min in healthy people. ${ }^{8,12,13}$ Blinking patterns appear to be associated with psychological conditions and/or certain systemic diseases..$^{8,13-15}$ There are several reports describing patients with systemic diseases as showing changes in blinking patterns; increased blinking rates in patients with schizophrenia ${ }^{1} 4$ or dry eye, ${ }^{8}$ and decreased blinking rates in patients with parkinsonism 13 or panic disorder. 5 Patients with schizophrenia, parkinsonism, or panic disorder were excluded from this study, but
Table 3 Multivariate regression analysis

\begin{tabular}{lrcc}
\hline Factor & $\begin{array}{c}\text { Regression } \\
\text { coefficient }\end{array}$ & $\begin{array}{c}\text { Standard-regression } \\
\text { coefficient }\end{array}$ & $P$ \\
\hline Tear lipid layer & 0.464 & 0.345 & $<0.0001$ \\
Tear breakup time & -0.147 & -0.392 & $<0.0001$ \\
Corneal sensitivity & 0.029 & 0.032 & 0.62 \\
Cotton thread test & 0.004 & 0.025 & 0.70 \\
Blinking rate & -0.003 & -0.035 & 0.58 \\
\hline
\end{tabular}

$R^{2}=0.36$

some with dry eye may have been included. In previous studies of diabetic patients, tear breakup time and the results of the Schirmer test were decreased, suggesting dry eye, and corneal sensitivity was decreased. ${ }^{5,16}$ In this study, tear breakup time and corneal sensitivity were also decreased in diabetic patients. Although patients with dry eye have been reported as having increased blinking rates, ${ }^{8}$ in this study patients with diabetes showed decreased blinking rates. Other factors apart from dry eye would tend to decrease blinking rates in patients with diabetes.

There are no previous reports evaluating the correlation between blinking rates and these ocular factors in patients with diabetes. In normal subjects, the 
blinking rate was significantly lower following anaesthetic administration (decreased corneal sensitivity), ${ }^{17}$ and there was a significant correlation between tear breakup time and the blinking rate. ${ }^{12}$ In this study, the blinking rates in patients with diabetes did not show any significant correlations with other ocular factors such as corneal sensitivity, tear breakup time, the status of lipid layer, or the result of cotton thread test (data not shown).

In this study, the average mean BT was $0.19 \pm 0.05 \mathrm{~s}$ in patients without and $0.20 \pm 0.05 \mathrm{~s}$ in those with diabetes. These results are essentially consistent with those of a previous report, ${ }^{8}$ which showed the average mean BT to be $0.20 \pm 0.04$ and $0.27 \pm 0.16 \mathrm{~s}$ in normal subjects and patients with dry eye, respectively. The IBT in patients with dry eye has been reported as significantly shorter. ${ }^{6}$ In patients with diabetes, the IBT was significantly longer in our study. A change in blinking rate would be induced by an IBT change.

The overall prevalence of superficial punctate keratopathy was $23.9 \%$ in the diabetic patients, similar to that in our previous report. ${ }^{5}$ We previously evaluated the relationship between the severity of diabetic keratoepitheliopathy and various ocular and systemic factors by multivariate regression analysis, and revealed that only the state of the tear lipid layer was significantly associated with the development of diabetic keratoepitheliopathy. ${ }^{5}$ In this study, the blinking rate was added as one of the ocular factors, and we re-evaluated the factors that contributed to the severity of superficial punctate keratopathy. The status of tear lipid layer and tear breakup time were significantly correlated with the index of superficial punctate keratopathy, but the blinking rate was not. Corneal sensitivity, hypothesized to be related to blinking, did not have any significant correlations with the severity of superficial punctate keratopathy. Among these ocular factors, tear-related factors including the status of tear lipid and tear breakup time are more important contributors to the severity of superficial punctate keratopathy than corneal sensitivity and blinking rate.

In conclusion, in patients with diabetes the IBT was prolonged, resulting in decreased blinking rate. However, the presence of superficial punctate keratopathy cannot be predicted from blinking patterns in diabetic patients.

\section{References}

1 Herse PR. A review of manifestations of diabetes mellitus in the anterior eye and cornea. Am J Optom \& Physiol Optics 1988; 65: 224-230.

2 Saini JS, Khandalavla B. Corneal epithelial fragility in diabetes mellitus. Can J Ophthalmol 1995; 30: 142-146.

3 Hatchell DL, Magolan JJ Jr, Besson MJ, Goldman AI, Pederson HJ, Schultz KJ. Damage to the epithelial basement membrane in the corneas of diabetic rabbits. Arch Ophthalmol 1983; 101: 469-471.

4 Schultz RO, Van Horn PL, Peters MA, Klewin KM, Schutten WH. Diabetic keratopathy. Trans Am Ophthalmol Soc 1981; 79: 180-199.

5 Inoue K, Kato S, Ohara C, Numaga J, Amano S, Oshika T. Ocular and systemic factors relevant to diabetic keratoepitheliopathy. Cornea 2001; 20: 798-801.

6 Miyata K, Sawa M, Nishida T, Mishima H, Miyamoto Y, Otori T. Classification of severity of superficial punctate keratitis. Jpn J Clin Ophthalmol (in Japanese) 1994; 48: 183-188.

7 Yokoi N, Takehisa Y, Kinoshita S. Correlation of tear lipid layer interference patterns with the diagnosis and severity of dry eye. Am J Ophthalmol 1996; 122: 818-824.

8 Tsubota K, Hata S, Okusawa Y, Egami F, Ohtsuki T, Nakamori K. Quantitative videographic analysis of blinking in normal subjects and patients with dry eye. Arch Ophthalmol 1996; 114: 715-720.

9 Sakamoto R, Bennett ES, Henry VA, Paragina S, Narumi T, Izumi $Y$ et al. The phenol red thread tear test: A crosscultural study. Invest Ophthalmol Vis Sci 1993; 34: 3510-3514.

10 Paschides CA, Kitsios G. Evaluation of tear break-up time, Schirmer's 1 test and rose bengal staining as confirmatory tests for keratoconjunctivitis sicca. Clin Exp Rheumatol 1989; 7: 155-157.

11 Cochet $\mathrm{P}$, Bonnet R. L'esthesiometrie corneenne: realisation et interet pratique. Bull Soc Ophthalmol Fr 1961; 61: 541-550.

12 Al-Abdulmunem M. Relation between tear break-up time and spontaneous blink rate. Int Contact Lens Clin 1999; 26: 117-120.

13 Karson CN, Burns RS, LeWitt PA, Calne DB, Wyatt RJ. Blink rates and disorders of movement. Neurology 1984; 34: 677-678.

14 Kleinman JE, Karson CN, Weinberger DR, Freed WJ, Berman KF, Wyatt RJ. Eye-blinking and cerebral ventricular size in chronic schizophrenic patients. Am J Psychiatry 1984; 141: $1430-1432$.

15 Kojima M, Shioiri T, Hosoki T, Sakai M, Bando T, Someya T. Blink rate variability in patients with panic disorder: new trial using audiovisual stimulation. Psychiatry Clin Neurosci 2002; 56: 545-549.

16 Dogru M, Katakami C, Inoue M. Tear function and ocular surface changes in noninsulin-dependent diabetes mellitus. Ophthalmology 2001; 108: 586-592.

17 Collins M, Seeto R, Campbell L, Ross M. Blinking and corneal sensitivity. Acta Ophthalmol 1989; 67: 525-531. 